\title{
Genome-scale reconstruction of metabolic network for a halophilic extremophile, Chromohalobacter salexigens DSM 3043
}

\author{
Özlem Ates, Ebru Toksoy Oner, Kazim Y Arga*
}

\begin{abstract}
Background: Chromohalobacter salexigens (formerly Halomonas elongata DSM 3043) is a halophilic extremophile with a very broad salinity range and is used as a model organism to elucidate prokaryotic osmoadaptation due to its strong euryhaline phenotype.

Results: C. salexigens DSM 3043's metabolism was reconstructed based on genomic, biochemical and physiological information via a non-automated but iterative process. This manually-curated reconstruction accounts for 584 genes, 1386 reactions, and 1411 metabolites. By using flux balance analysis, the model was extensively validated against literature data on the C. salexigens phenotypic features, the transport and use of different substrates for growth as well as against experimental observations on the uptake and accumulation of industrially important organic osmolytes, ectoine, betaine, and its precursor choline, which play important roles in the adaptive response to osmotic stress.

Conclusions: This work presents the first comprehensive genome-scale metabolic model of a halophilic bacterium. Being a useful guide for identification and filling of knowledge gaps, the reconstructed metabolic network iOA584 will accelerate the research on halophilic bacteria towards application of systems biology approaches and design of metabolic engineering strategies.
\end{abstract}

\section{Background}

Extreme environments, generally characterized by abnormal temperature, $\mathrm{pH}$, pressure, salinity, toxicity and radiation levels, are inhabited by various organisms - extremophiles - that are specifically adapted to these particular conditions. Studies on these microorganisms has led to the development of important molecular biology techniques such as polymerase chain reaction (PCR) $[1,2]$ and hence further research has been largely stimulated by the industry's interest on the fact that the survival mechanisms of these microorganisms could be transformed into valuable applications ranging from wastewater treatment to the diagnosis of infectious and genetic diseases [3].

Halophilic microorganisms are extremophiles that are able to survive high osmolarity in hypersaline conditions either by maintenance of high salinity in their cytoplasm

\footnotetext{
* Correspondence: kazim.arga@marmara.edu.tr

Department of Bioengineering, Marmara University, 34722, Istanbul, Turkey
}

or by intracellular accumulation of osmoprotectants such as ectoine and betaine [4]. C. salexigens is a halophilic Gammaproteobacterium of the family Halomonadaceae with a versatile metabolism allowing not only fast growth on a large variety of simple carbon compounds as its sole carbon and energy source but also resistance to saturated and aromatic hydrocarbons and heavy metals $[5,6]$. C. salexigens with the ability to grow over a wide range of salinities $[0.5-4 \mathrm{M} \mathrm{NaCl}]$ has been the most euryhaline of the bacteria [7] and to understand the osmoregulatory mechanisms in halophilic bacteria, it has been used as a model organism [5,7-9]. Moreover, C. salexigens has also many promising biotechnological applications as a source of compatible solutes, salt-tolerant and recombinant enzymes, biosurfactants and exopolysaccharides [10].

Genome sequence of extremophiles, such as sulphatereducing archaeon Archaeaglobus fulgidus [11], halophilic archaeon Halobacterium species NRC-1 [12] and acidophilic bacterium Acidithiobacillus ferrooxidans [13]
Ciomed Central

() 2011 Ates et al; licensee BioMed Central Ltd. This is an Open Access article distributed under the terms of the Creative Commons Attribution License (http://creativecommons.org/licenses/by/2.0), which permits unrestricted use, distribution, and reproduction in any medium, provided the original work is properly cited. 
have been reported earlier. Since the publication of the genome of C. salexigens DSM 3043 [14] the biological knowledge about this strain has significantly increased and various methods that allow the genomic analysis and genetic manipulation have been developed $[15,16]$. On the other hand, systematic analysis of its metabolic and biotechnological capacities have not been performed yet. This is, at some level, due to the lack of an in silico comprehensive metabolic model that enables the integration of canonical experimental data in a coherent fashion.

Metabolic reconstruction is non-automated and iterative decision-making process through which the genes, enzymes, reactions and metabolites that participate in the metabolic activity of a biological system are identified, categorized and interconnected to form a network [17]. The reconstruction process has been reviewed conceptually in literature [17-22] and, recently, a standard operating protocol giving a detailed overview of the necessary data and steps has been published [23]. To date, genomescale metabolic reconstructions for more than 50 organisms have been published and this number is expected to increase rapidly. Therefore, the need for developing automated, or at least semi-automated, ways to reconstruct metabolic networks is growing. A limited number of software tools, such as Pathway tools [24], metaSHARK [25], Simpheny (Genomatica), which aim at assisting and facilitating the reconstruction process are available. However, recent reviews $[18,26]$ highlight current problems with genome annotations and databases, which make automated reconstructions challenging and thus they require manual evaluation. Genome-scale metabolic reconstructions have been successfully applied to several organisms across eukaryotic (e.g., Saccharomyces cerevisiae [21,27-29], human [30], Arabidopsis thaliana [31]), prokaryotic (e.g., Escherichia coli [32-34], Bacillus subtilis [35], Helicobacter pylori [36,37], Lactococcus lactis [38], Staphylococcus aureus [39,40], Clostridium acetobutylicum [41], Pseudomonas putida [42], Pseudomonas aeruginosa [43], Geobacter metallireducens [44], Corynebacterium glutamicum [45]), and archaeal (e.g., Methansoarcina barkeri [46], Halobacterium salinarum [47] species). Being a useful guide for identification and filling of knowledge gaps, these metabolic networks have been used toward simulation of the cellular behavior under different genetic and physiological conditions, contextualization of highthroughput data, directing hypothesis driven discovery, interrogation of multi-species relationships and topological analysis (See [17] for an extensive review).

Here, a genome-scale reconstruction of C. salexigens DSM 3043's metabolism was established based on genomic, biochemical and physiological information. Being the first comprehensive metabolic model of a halophilic bacterium, it was labeled as $i$ OA584 following the naming convention proposed by [33]. The predictive potential of the model was validated not only against literature data on the in vivo $C$. salexigens phenotypic features, the transport and use of different substrates but also against experimental observations on the choline - betaine and ectoine synthesis pathways which are important parts of the osmoadaptation mechanism.

\section{Methods}

\section{Genome Annotation}

The complete genome sequence of $C$. salexigens DSM 3043 has been assembled in 2005 by the Joint Genome Institute [14] and gene annotations are available online at the web-sites of Computational Biology at ORNL [48] and Joint Genome Institute [14], which represent computational platforms enabling the corresponding enzymes in addition to gene catalog. C. salexigens DSM 3043 genome size is $3.696 \mathrm{Mb}$ with 3352 candidate protein-encoding gene models.

\section{Reconstruction Process}

For the reconstruction of a genome-scale metabolic network of the halophilic bacterium C. salexigens DSM 3043, a non-automated but iterative decision-making process is designed based on the conceptual reviews $[18,19,22]$ and published protocol [23]. In the first stage, a draft reconstruction was built from gene-annotation data $[48,49]$ coupled with information from online databases, which link genes to functional categories and help bridge the genotype-phenotype gap. For the association of the enzymes to the biochemical reactions, biochemical information databases KEGG [50], BiGG [51], ExPASy [52], BioCyc [53] and BRENDA [54], which provide comprehensive information on enzymes and biochemical reactions, were employed to extract metabolic reactions, their stoichiometry and thermodynamic constraints (i.e. reversibility). As a result of the first stage, an initial catalog of gene-enzyme-reaction associations was prepared. In the second stage, the draft reconstruction was refined semiautomatically through gap analysis. Using the draft catalog, the stoichiometric matrix, the reaction and metabolite adjacency matrices [55] were constructed, metabolic maps were drawn and topological analysis $[56,57]$ was performed. Analysis of the preliminary version of the network indicated the occurrence of metabolites not connected with the overall metabolic network, i.e. the presence of dead-end metabolites. The resulting shortage was overcome mostly by manually searching biochemical information databases [50-54] and carrying out a comprehensive literature survey on metabolisms of $C$. salexigens $[5-9,16,58,59]$. In the last stage, the biomass formation and transport reactions, which describe the intra- and extracellular exchange of metabolites, were added to the metabolic network predominantly based on the experimental 
evidence on phenotypic characterization of the strain $[5-9,16,58,59]$. The reconstructed metabolic network was automatically converted into a mathematical model that could be analyzed through constraint-based approaches, and was validated through comparison of model predictions with phenotypic data.

\section{Constraint-based Modeling}

The interconnectivity of metabolites in a biochemical reaction network can be represented by a set of equations defining the stoichiometric conversion of substrates into products [60]. The reconstructed metabolic network was represented by a stoichiometric matrix, $\mathrm{S}(m \times n)$ where $m$ is the number of metabolites and $n$ is the number of reactions. The corresponding entry in the stoichiometric matrix, $\mathrm{S}_{i j}$, represents the stoichiometric coefficient for the participation of the $\mathrm{i}^{\text {th }}$ metabolite in the $\mathrm{j}^{\text {th }}$ reaction. A constraint-based optimization framework, Flux Balance Analysis (FBA) [61,62], was then recruited to solve the linear programming problem under steady-state criteria represented by the equation () where $\mathrm{v}$ is a vector of reaction fluxes. Since the optimization problem belongs to an under-determined system, there exist multiple solutions. To find a particular solution for reaction fluxes, the cellular objective of producing the maximum amount of biomass constituents was optimized [63]. The employment of optimal growth assumption has allowed successful calculation of phenotypic behaviour in FBA of reconstructed metabolic models of several microorganisms [34-36,38, 40-42,46,47], suggesting that their metabolic networks have evolved for the optimization of the specific growth rate under several carbon source limiting conditions. Constraints need to be imposed on the system in the form of inequality () where $\alpha$ and $\beta$ are the lower and upper limits placed on each reaction flux, respectively. The constraint-based optimization problem was solved using MATLAB 7.4 (The Mathworks, Inc.).

\section{Biomass Formulation}

No thorough biomass composition has been published for $C$. salexigens. The use of a generic biomass formation reaction in FBA simulations was previously tried and led to successful predictions $[34,39,64]$. Hence, based on the experimental evidences on genome similarity [7], phylogenetic classification and results from the comparative analysis of the $C$. salexigens metabolic network with other published reconstructed networks [27,34,35,39, $42,43,46,47]$, the relative production of metabolites required for growth was taken from the published composition of E. coli iAF1260 [34].

\section{Flux Variability Analysis}

The flux variabily analysis was performed [65] to observe the alternate optimal flux distributions. Briefly, the optimal value of the objective function was calculated by FBA simulation; then, with the objective function fixed at the optimal value, for each reaction the maximum and minimum possible fluxes were computed. The two values calculated for each reaction characterize its variability.

\section{Results And Discussion}

\section{Metabolic Reconstruction Process}

Based on the conceptual reviews [19,18,22] and published protocol [23], a non-automated but iterative three-stage process was designed to reconstruct a genome-scale metabolic network of the halophilic bacterium C. salexigens DSM 3043.

In the first stage, a draft catalog of gene-enzyme-reaction associations was prepared via coupling genome annotation data $[48,49]$ with biochemical information databases [50-54]. The genome annotation resources for C. salexigens $[48,49]$ not only include genetic information such as genome position, coding region, locus tag, gene product function, but also represent assignments of gene products to PRIAM categories, COG functional groups, KEGG orthologies and pathways, and Enzyme Commission (EC) numbers. All these information were assembled and analyzed manually to identify candidate metabolic functions. In the first step, the pathway databases, namely KEGG [50] and BiGG [51], were systematically searched for the associations of the metabolic reactions to the enzymes. At this step, KEGG pathway assignments and EC numbers, which represent a hierarchical classification of enzymatic reactions and are commonly utilized as identifiers of enzymes in the analysis of complete genomes, played important role in bridging the genomic repertoire of gene models to the chemical repertoire of metabolic pathways. However, several EC numbers were assigned to signaling or regulatory proteins, whose functions are not normally considered in metabolic reconstructions. For instance, Csal2070 gene was assigned for a repressor protein LexI (EC 3.4.21.88) functioning in SOS regulation. Therefore, these assignments were carefully checked and not included in the draft reconstruction. Another important point to be emphasized is the incompleteness of pathway databases. Although very high percentages (66.6\%) of the enzymes were associated with the reactions, there were missing reactions that were not represented in these databases. In the second step, enzyme information databases, namely ExPASy [52], BioCyc [53] and BRENDA [54] were explored to include the missing reactions to the model. Since EC numbers were known from previously obtained gene-annotation data, enzymes could be connected with accurate metabolic reactions. For example, the reactions for carbonyl reductase (EC 1.1.1.184), malate synthase (EC 2.3.3.9) and creatinase 
(EC 3.5.3.3) were obtained from ExPASy, BRENDA and BioCyc databases, respectively. The outcome of the first stage was an initial catalog of gene-enzyme-reaction associations.

Second stage comprised of semi-automatically refinements of the draft reconstruction through gap analysis. Using the draft catalog of gene-enzyme-reaction associations, the stoichiometric matrix, the reaction and metabolite adjacency matrices were constructed, metabolic maps were drawn and topological analysis was performed [55,56] Analysis of the preliminary version of the network indicated the occurance of metabolites not connected with the overall metabolic network, i.e. the presence of dead-end metabolites. Their presence might be due to a misassignment of a gene function or to missing reactions linking these metabolites with the overall network. The resulting shortage was overcome mostly by manually searching other biochemical information databases, namely ExPASy [52], BioCyc [53] and BRENDA [54]. In addition for these enzyme-reaction associations, the required information was obtained from literature. For instance, in the utilization pathway of tagatose, tagatose-6-phosphate kinase reaction (EC 2.7.1.144) was present in the model; but, an essential intermediate step, i.e. the formation reaction of tagatose 6 -phosphate from tagatose, was missing in the model. Subsequently, tagatose kinase reaction (EC 2.7.1.101) was included to the model. In some cases, gap analysis indicated the lack of numerous steps in several pathways. For example, in arabinose metabolism 5 additional metabolic reactions (EC 1.1.1.46, EC 3.1.1.15, EC 4.2.1.25, EC 4.2.1.43 and EC 1.2.1.26) were required to link dead-end metabolites to the metabolic model. At this stage, stoichiometrically unbalanced reactions were also checked. Normally, there are two common errors causing unbalanced reactions [23]: Missing proton and/ or water, or when the stoichiometric coefficient of at least one metabolite is wrong. All the metabolic reactions were tested for mass and charge balancing and several reactions required corrections. For example, in the reaction catalyzed by glucokinase (EC 2.7.1.2, Csal0935), which was obtained from KEGG [50], a proton was missing.

In the last stage, the reconstructed metabolic network was automatically converted into a mathematical model that could be analyzed through constraint-based approaches, and was validated through comparison of model predictions with phenotypic data. The biomass formation and transport reactions, which describe the intra- and extracellular exchange of metabolites, were added to the metabolic network predominantly based on the experimental evidence [5-9,16,58,59] on phenotypic characterization of the strain and then FBA simulations on various carbon sources were performed to verify the model. For example, uptake of macro nutrients (e.g., amino acids, sucrose, glucose), secretion of by-products (e.g., lactate, ammonia, betaine), and exchange of free compounds (water, carbon dioxide, oxygen) were added since they represent essential cellular inputs and outputs. The metabolic model was updated iteratively using the above procedure until the in silico phenotypic characterizations were completely represented by the simulation results.

\section{Characteristics of the Reconstructed Metabolic Network of C. salexigens}

The reconstruction process resulted in a metabolic network that consisted of 1387 metabolic reactions including biomass reaction and 1411 metabolites (Additional File 1). The model is composed of 876 enzymatic reactions, 510 transport reactions; 920 intracellular and 491 extracellular metabolites and throughout the reconstruction process, 584 protein-encoding gene models have been assigned to the metabolic reactions (Table 1). For $97.7 \%$ of all enzymatic reactions, a corresponding gene-enzyme-reaction association has been assigned in the model.

A large amount of enzymes, which were included by the metabolic model, were monofunctional (80.65\%) whereas the rest were multifunctional accepting several different substrates. Therefore, the published genome for their corresponding genes were carefully checked during reconstruction process in order not to lead to false gene-enzyme-reaction associations in the reconstructed genome-scale metabolic model.

The enzymes included in $i$ OA584 were divided into 12 main categories based on their functional roles (Figure 1A). The transport category was found to be the subsystem with the highest number of enzymes (40\%), highlighting the importance of cellular transport for $C$. salexigens. Most of the transport reactions were included into the network based on physiological data and the abundance of transport reactions agrees well with the experimental findings that this organism has an excellent adaptation to osmotic stress [8] and is able to

\section{Table 1 Network characteristics of the reconstructed metabolic network of C. salexigens}

\begin{tabular}{lc}
\hline Protein-encoding gene models & 584 \\
Metabolites & 1411 \\
Intracellular metabolites & 920 \\
Extracellular metabolites & 491 \\
Reactions & 1386 \\
Enzymatic reactions & 876 \\
Transport fluxes & 510 \\
Reactions with protein-encoding gene model assignments & 886 \\
Enzymatic reactions & 856 \\
Transport fluxes & 30
\end{tabular}


A

Pathway Distribution

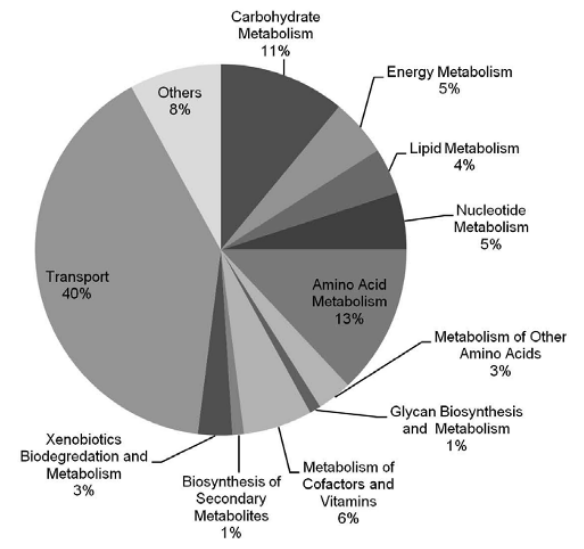

B

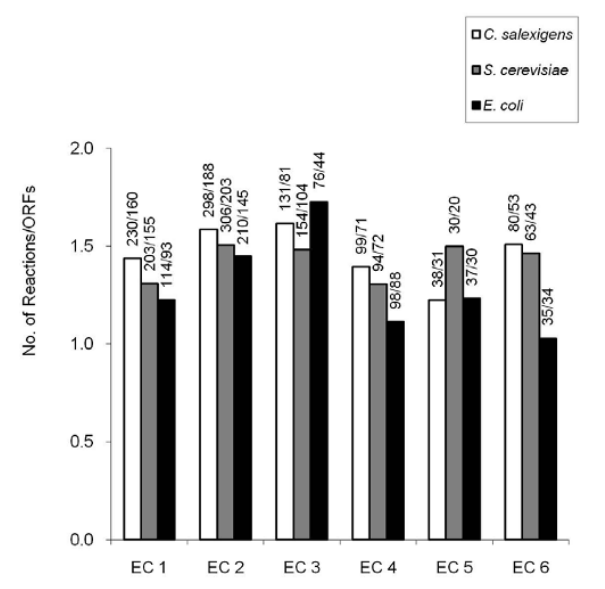

Figure 1 Characteristics of the reconstructed metabolic network iOA584. A: Distribution of the 12 main metabolic pathways in iOA584. B Comparison of the distribution of enzyme classes in C. salexigens, E. coli and S. cerevisiae.

utilize various carbon sources as sole energy source. However, the high number of transport reactions with no gene assignment (94\% of transport reactions) in C. salexigens points to the fact that further work is needed to characterize the mechanisms and genetic machinery involved in the transport of molecules in halophilic bacteria. For example, although the halophilic bacterium is known to be able to utilize various carbon sources as sole energy source, only 4 genes (Csal0010, Csal1144, Csal0500, and Csal1728) were associated with sugar transport mechanisms in the annotated genome of $C$. salexigens [38]. In addition, only one of them (Csal0010) has been associated with an enzyme (EC.2.7.8.20) in BioCyc [53]. Deciphering the transport phenomena in halophilic bacteria is an important issue, since understanding the osmoprotectant uptake mechanisms in natural environments is a key point in achieving an efficient osmoadaptation. Therefore, for further studies, a detailed biophysical classification of the 342 candidate gene models related to the transport mechanism was presented (See Additional File 2 for the complete list of genes and their annotations).

Moreover, C. salexigens is known for its capability to utilize many amino acids as a carbon and nitrogen source [5,6,59]. The presence of high number of enzymes (13\%) in the amino acid metabolism is in agreement with the fact that the de novo synthesis pathways for all 20 amino acids are present in C. salexigens' genome $[14,48]$. To validate in silico amino acid utilization as a carbon and nitrogen source, FBA simulations were carried out and growth on all of the 20 amino acids were obtained. For instance, at a specific uptake rate $(1 \mathrm{mmol} / \mathrm{gDW} / \mathrm{h})$ of isoleucine, growth rate was calculated as $0.129 \mathrm{~h}^{-1}$. Additionally, glycan biosynthesis and metabolism; and biosynthesis of secondary metabolites have the lowest number of enzymes (1\%).

Throughout the reconstruction process, 584 proteinencoding gene models have been assigned to the metabolic reactions. The distribution of the ratios of number of reactions per number of gene models in each enzyme class $[27,32]$ was investigated in the reconstructed model $i$ OA584 (Figure 1B). In the metabolic network of C. salexigens, hydrolases (EC 3) were positioned primarily, followed by transferases (EC 2), ligases (EC 6), oxidoreductases (EC 1), lyases (EC 4), and isomerases (EC 5). Hence ligases and transferases were less substrate specific than the other enzyme classes in C. salexigens, as in the case of E. coli [32], whereas in S. cerevisiae isomerases and transferases were found to be the least substrate-specific enzyme classes [27].

Related species of the same domain share a substantial amount of conserved reactions for essential biological processes [66-68]. The metabolic network $i$ OA584 was also compared with previous metabolic models from different domains $[27,34,35,39,42,43,46,47]$ to identify the conserved reactions in $i$ OA584. As expected, highest number of metabolic reactions were shared by $E$. coli (iAF1260) with 320 reactions, P. aeruginosa (iMO1056) with 309 reactions and $P$. putida (iJN746) with 282 reactions. Number of shared metabolic reactions for $S$. cerevisiae, S. aureus N315, B. subtilis, and H.salinarium were obtained as 274, 265, 260, and 221, respectively; while $C$. salexigens and $M$. barkeri association indicated the lowest number with 205 metabolic reactions. 
The distribution of the reactions for C. salexigens, E. coli and the eukaryote S. cerevisiae (Figure 2A) indicated an interior set of 228 reactions in all of the three metabolic models with the following pathway distribution; 95 from amino acid, 53 from carbohydrate, 52 from metabolism of cofactors and vitamins, 43 from nucleotide, 27 from energy and 18 from lipid metabolism. A number of reactions were found to be involved in more than one pathway such as reactions catalyzed by alcohol dehydrogenase (ADH) enzymes (EC 1.1.1.1) that can be found in carbohydrate, lipid and amino acid metabolisms in agreement with literature [69]. 37.3\% of the total reactions were unique to $C$. salexigens $i \mathrm{OA} 584$ most of which were from amino acid (38 reactions) metabolism followed by carbohydrate metabolism (31 reactions). Comparison of the distribution of metabolic reactions for $C$. salexigens, $P$. putida and $P$. aeruginosa (Figure 2B) showed a higher interior set with 250 reactions as expected, since species of the same domain share a substantial amount of conserved reactions for essential biological processes [68]. A similiar pathway distribution was observed whereas most of the reactions were involved in amino acid, carbohydrate metabolism and lipid metabolism with 118, 89 and 57 reactions, respectively.

\section{Capabilities of the metabolic network - Phenotypic characterization in silico}

One of the major requirements for a reconstructed network is its compatibility with the physiology of the organism which in turn is highly essential when using the model in understanding the diverse mechanisms of the organism. In the present study, in silico phenotypic characterization constitutes an essential step of the reconstruction process. At the last stage of the reconstruction process, FBA simulations were performed with various growth media to test for incapabilities of the

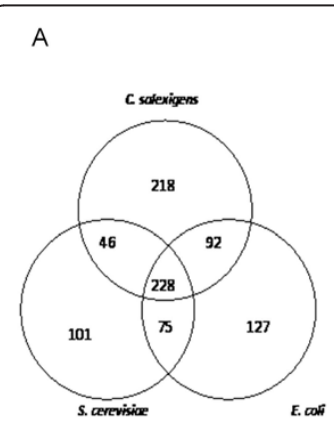$$
\text { B }
$$

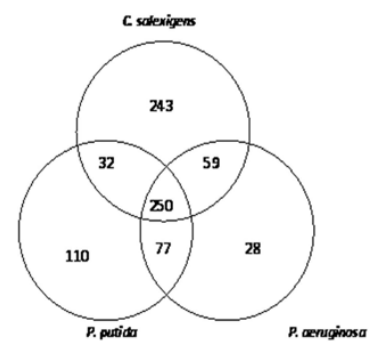

Figure 2 Comparison of the reconstructed metabolic network with previous metabolic models from different domains. A: The distribution of reactions in C. salexigens, E. coli and S. cerevisae B: The distribution of reactions in C. salexigens, $P$. putida and P.aeruginosa model in representing the phenotypic features in literature $[5-9,16,58,59]$ (Table 2). For example, the metabolic model $i$ OA584 was not able to utilize galactitol, tagatose, xylose, erythritol, arabinose, malonate, propionate and glycerate due to the absence of several exchange and enzymatic reactions. These shortages were resolved via manual searching of biochemical information databases [52-54] and by addition of 13 reactions (EC 1.1.1.16, EC 1.1.1.175, EC 1.1.1.46, EC 1.2.1.15, EC 1.2.1.26, EC 3.1.1.15, EC 2.7.1.101, EC 2.7.1.27, EC 2.7.2.15, EC 4.2.1.25, EC 4.2.1.43, rxn978 and rxn1314) into the network. The metabolic network was updated until the complete in silico phenotypic characterization was achieved.

The resultant metabolic model $i \mathrm{OA} 584$ has the ability to verify reported $C$. salexigens phenotypic features [5-9,16,58,59] through in silico FBA simulations. C.salexigens is able to grow aerobically and has ability for anaerobic respiration with nitrate. This microorganism is catalase and citrate positive, oxidase negative. Nitrate can be reduced to nitrite in contrast nitrite cannot be reduced $[5,6,59]$. The in silico aerobic and anerobic growth simulations were performed with biomass as the objective function at a specific glucose uptake rate of 3 $\mathrm{mmol} / \mathrm{gDW} / \mathrm{h}$ and for anaerobic respiration with 1 $\mathrm{mmol} / \mathrm{gDW} / \mathrm{h}$ nitrate as an electron acceptor instead of $\mathrm{O}_{2}$. The growth rates were determined as $0.1934 \mathrm{~h}^{-1}$ and $0.0645 \mathrm{~h}^{-1}$ for aerobic and anaerobic conditions, respectively. As such, catalase, citrate, urease activities and nitrate reduction simulations were also consistent with literature data (Table 2). Acetoin, indole, lysine decarboxylase, ornithine decarboxylase and phenylalanine deaminase could not be produced by $C$. salexigens $i$ OA584 as also reported in vivo [6]. Literature data on the transport and use of 59 different substrates were also verified in silico by fixing the externally transport reaction of fluxes $(3-10 \mathrm{mmol} / \mathrm{gDW} / \mathrm{h})$ and investigating the associated utilization reaction fluxes and objective function biomass flux to assess a positive growth. For example, $1 \mathrm{mmol} / \mathrm{gDW} / \mathrm{h}$ uptakes of fructose and sucrose resulted in growth rates of $0.1290 \mathrm{~h}^{-1}$ and $0.0646 \mathrm{~h}^{-1}$, respectively.

Additionally, the FBA simulations were performed in order to validate experimental growth rate values with glucose as the only carbon source in chemically defined media which were reported [70]. Experimental and in silico growth fluxes for batch cultivation of $C$. salexigens at varying glucose uptake rates ( 3.193 - $3.751 \mathrm{mmol} /$ $\mathrm{gDW} / \mathrm{h}$ ) were illustrated in Figure 3 . Whereas a higher growth rate was predicted for $3.193 \mathrm{mmol} / \mathrm{gDW} / \mathrm{h}$, simulations were in significant agreement with the experimental data with as low as 1.5 to $2.5 \%$ errors for the other glucose concentrations $(3.307,3.478$ and 3.751 $\mathrm{mmol} / \mathrm{gDW} / \mathrm{h}$ ). 
Table 2 In silico predictions of the phenotypic features

\begin{tabular}{|c|c|c|c|}
\hline Phenotype & $\begin{array}{c}\text { (in vivo/in } \\
\text { silico) }\end{array}$ & Related Enzymes & Related Reactions \\
\hline Catalase activity & $(+/+)$ & Catalase (1.11.1.6) & rxn87 \\
\hline Citrate activity & $(+/+)$ & citrate synthase (2.3.3.1) & $r \times n 328$ \\
\hline Urease activity & $(+/+)$ & Urease (3.5.1.5) & rxn610 \\
\hline Nitrate reduction & $(+/+)$ & $\begin{array}{c}\text { Nitrate reductase } \\
\quad(1.7 .99 .4)\end{array}$ & rxn216-rxn218 \\
\hline Substrate & $\begin{array}{l}\text { (in vivo/in } \\
\text { silico) }\end{array}$ & Transport Reactions & Utilization Reactions \\
\hline Acetate & $(+/+)$ & rxn964 & rxn527, rxn559, rxn817 \\
\hline Adonitol & $(-/-)$ & - & - \\
\hline Glycine Betaine & $(+/+)$ & rxn1112, rxn1113 & $r \times n 136, r \times n 137, r \times n 243$ \\
\hline Butyrate & $(-/-)$ & - & - \\
\hline Caprylate & $(-/-)$ & - & - \\
\hline Cellobiose & $(-/-)$ & - & - \\
\hline Choline & $(+/+)$ & rxn1004, rxn1005 & rxn83, rxn107, rxn580 \\
\hline Citrate & $(+/+)$ & rxn1007 & rxn328, rxn702, rxn726, rxn727 \\
\hline Creatine & $(-/-)$ & - & - \\
\hline D-fructose & $(+/+)$ & rxn1079, rxn1080 & rxn62, rxn337, rxn433, rxn781 \\
\hline D-galactose & $(+/+)$ & rxn1091 & rxn53 \\
\hline D-glucose & $(+/+)$ & rxn877, rxn881, rxn882 & rxn82, rxn422 \\
\hline DL-glycerate & $(+/+)$ & rxn1114 & rxn64 \\
\hline $\begin{array}{l}\text { DL- } \alpha- \\
\text { aminobutyrate }\end{array}$ & $(-/-)$ & - & - \\
\hline D-mannitol & $(+/+)$ & rxn1173 & rxn33, rxn62 \\
\hline D-mannose & $(+/+)$ & rxn1161 & rxn33, rxn781 \\
\hline D-melibiose & $(-/-)$ & - & - \\
\hline D-raffinose & $(-/-)$ & - & - \\
\hline D-ribose & $(+/+)$ & rxn1274 & rxn418 \\
\hline D-sorbitol & $(+/+)$ & rxn1284 & rxn17 \\
\hline L-tartrate & $(+/+)$ & rxn1312 & $r \times n 729$ \\
\hline D-trehalose & $(+/+)$ & rxn1327, rxn1328 & rxn562 \\
\hline $\begin{array}{l}\text { Dulcitol } \\
\text { (galactitol) }\end{array}$ & $(+/+)$ & rxn1101 & rxn53 \\
\hline D-xylose & $(+/+)$ & rxn1385 & $r \times n 22$ \\
\hline Erythritol & $(+/+)$ & rxn1060 & $r \times n 427$ \\
\hline Ethanol & $(+/+)$ & rxn1065 & $r \times n 2$ \\
\hline Fumarate & $(+/+)$ & rxn1081 & rxn155, rxn156, rxn158 - rxn162, rxn720, rxn753 - rxn755 \\
\hline Galactosamine & $(-/-)$ & - & - \\
\hline Gluconolactone & $(-/-)$ & - & - \\
\hline Glutamate & $(+/+)$ & rxn1109 & rxn763, rxn854 \\
\hline Glycerol & $(+/+)$ & rxn1116 & $r \times n 1, r \times n 429$ \\
\hline Glycine & $(+/+)$ & rxn1118 & rxn182, rxn183, rxn257, rxn258, rxn605, rxn695, rxn802, rxn848, rxn859 \\
\hline Inulin & $(-/-)$ & - & - \\
\hline Lactate & $(-/-)$ & - & - \\
\hline L-alanine & $(-/-)$ & - & - \\
\hline L-arabinose & $(+/+)$ & rxn978 & rxn52 \\
\hline L-arginine & $(+/+)$ & rxn979 & rxn275, rxn616, rxn661, rxn753, rxn807 \\
\hline L-asparagine & $(+/+)$ & rxn981 & rxn597 \\
\hline L-fucose & $(-/-)$ & - & - \\
\hline L-glutamine & $(+/+)$ & rxn1103 & rxn175, rxn184, rxn389, rxn699, rxn806, rxn863, rxn868 - rxn872 \\
\hline L-lysine & $(+/+)$ & rxn1153 & rxn104, rxn814 \\
\hline L-methionine & $(-/)$ & - & - \\
\hline L-ornithine & $(+/+)$ & $r \times n 1209$ & $r \times n 267, r \times n 268, r \times n 750$ \\
\hline L-proline & $(+/+)$ & rxn1248 & rxn195, rxn803 \\
\hline
\end{tabular}


Table 2 In silico predictions of the phenotypic features (Continued)

\begin{tabular}{|c|c|c|c|}
\hline L-rhamnose & $(-/-)$ & - & - \\
\hline L-serine & $(+/+)$ & rxn1290 & 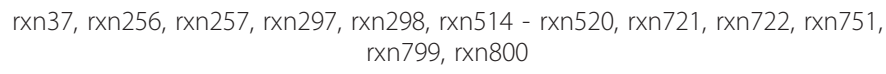 \\
\hline L-threonine & $(-/-)$ & - & - \\
\hline L-valine & $(-/-)$ & - & - \\
\hline Malate & $(+/+)$ & rxn1155 & rxn46, rxn47, rxn84, rxn85, rxn720 \\
\hline Malonate & $(+/+)$ & rxn1156 & rxn122 \\
\hline Maltose & $(+/+)$ & rxn1157 & rxn587 \\
\hline Meso-inositol & $(+/+)$ & $r \times n 1170$ & rxn96 \\
\hline Oxalate & $(-/-)$ & - & - \\
\hline Propionate & $(+/+)$ & $r \times n 1249$ & rxn433 \\
\hline Putrescine & $(-/-)$ & - & - \\
\hline Sarcosine & $(-/-)$ & - & - \\
\hline Succinate & $(+/+)$ & rxn1305 & rxn97, rxn98, rxn156, rxn159, rxn160, rxn163, rxn700, rxn841 \\
\hline Sucrose & $(+/+)$ & rxn883, rxn884, rxn885 & rxn337 \\
\hline Tagatose & $(+/+)$ & rxn1314 & rxn39, rxn439 \\
\hline$\alpha$-lactose & $(-/-)$ & - & - \\
\hline
\end{tabular}

Since the flux distribution of overall network map might be useful in investigating and improving FBA analysis, Omics Viewers Tool of BioCyc [53] was used to illustrate in silico flux distribution in C. salexigens metabolic pathways. Reaction flux data and gene information were provided for Omics Viewer to generate overall diagram colorized with flux data. The details of the connectivity aspects of the reconstructed metabolic network (Additional File 3), the overall map of the reconstructed network and its detailed batch images obtained were also supplemented (Additional File 4).

\section{Case study on osmoadaptation}

Generally, halophiles can adapt to the saline environment by either intracellular accumulation of salts, or exclusion of salts and production or accumulation of

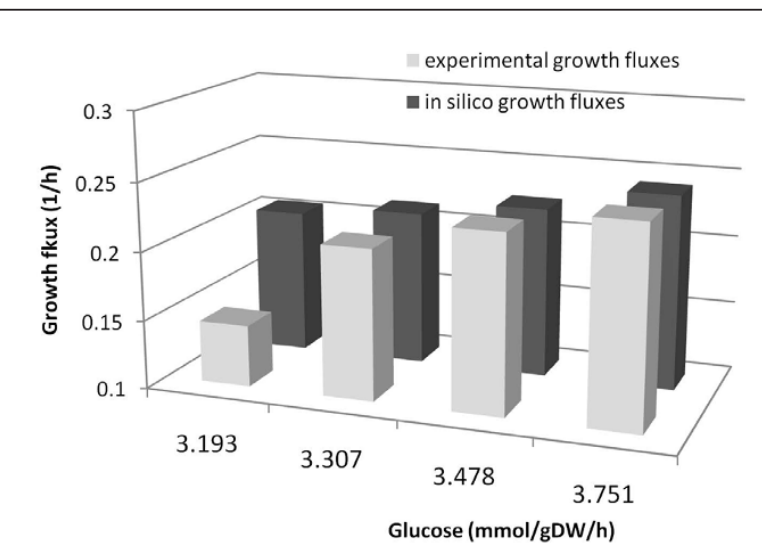

Figure 3 Experimental and in silico growth fluxes for batch cultivation of C.salexigens when glucose uptake rate was varying between $3.193-3.751 \mathrm{mmol} / \mathrm{gDW} / \mathrm{h}$. different classes of organic solutes (osmoprotectants) $[71,72]$. C. salexigens has been used comprehensively as a model organism in osmoadaptation studies due to its ability to grow over a wide range of salinities [6-8]. Osmoadaptation in C. salexigens is mainly achieved by de novo synthesis of two compatible solutes, namely ectoine and hydroxyectoine, which are of industrial and biological interest due to their biostabilizing properties. In addition, when these solutes are provided externally, C. salexigens accumulates other osmoprotectants such as choline and glycine betaine. Besides the betaine exchange that is common in bacteria, the rarely encountered betaine biosynthesis pathway from choline has been characterized in C. salexigens to some extend at the biochemical level $[5,8,59,73,74]$. Further research on the genes and metabolic pathways responsible for the biosynthesis of compatible solutes will not only find numerous applications in biomedicine, agriculture, food and fermentation industries but also expand our knowledge on the prokaryotic adaptation mechanisms to abiotic stresses like high salinity [72].

Via integration of data from in vitro metabolic and genetic analyses, in further studies, the presented genome scale model iOA584 could be used to elucidate osmoadaptation mechanisms and to design strategies (i.e. optimizing culture media, genetical engineering of the microorganism) for optimum production of compatible solutes such as ectoine, which has industrial applications for cosmetics and dermopharmacy and is widely used in stabilizing enzymes for molecular biology.

Here, C. salexigens iOA584 was used to simulate the experimental observations on osmoadaptation of C. salexigens, in order to demonstrate that the model could be used for further studies in understanding the metabolic 
pathways behind osmoadaptation and to design or improve the adaptation mechanisms in extromophiles.

In $C$. salexigens, the osmoprotectant betaine is synthesized from its precursor choline in two steps (Figure 4). In the first step, choline is converted into betaine aldehyde by membrane-bound choline dehydrogenase (EC 1.1.99.1, Csal1514) or by a ferredoxin-dependent choline monooxygenase (EC 1.14.15.7, Csal2455). Then, betaine aldehyde dehydrogenase (EC 1.2.1.8, CsaI1515) catalyzes the conversion of betaine aldehyde to betaine in the second step. Previously, Canovas and coworkers (1998) have investigated the transport of choline and its conversion to the osmoprotectant compound glycine betaine in C. salexigens. They reported that the growth of $C$. salexigens (with glucose as the sole carbon source) was stimulated by the presence of choline and that the presence of betaine had an inhibitory effect on the intracellular oxidation of choline.

For validation of the model's predictive potential, in silico model simulations of the choline - betaine pathway of the osmoadaptation mechanism were compared with these experimental observations [5,7-9,59]. FBA simulations were performed with biomass as the objective function and 1 to $3 \mathrm{mmol} / \mathrm{gDW} / \mathrm{h}$ glucose uptake rate (Figure 4). Via restriction of uptake of exogeneous choline to various values between 1 to $2 \mathrm{mmol} / \mathrm{gDW} / \mathrm{h}$, monotonic increase in the biomass flux (from 0.065 to $0.129 \mathrm{~h}^{-1}$ ) and in betaine production flux (from 0.45 to $0.89 \mathrm{mmol} / \mathrm{gDW} / \mathrm{h}$ ) were observed; hence stimulation of growth by the presence of choline was predicted, which is in agreement with the reported experimental observations [58]. It is known that the resulting solution of FBA especially when applied to genome scale models is normally not unique [65]. Therefore, the flux variability analysis was performed to observe the alternate optimal flux distributions in FBA simulations. Results showed that the fluxes are in general not affected since the range of variabilities for each flux were lower than $0.1 \%$.

Due to the high industrial and biological importance of ectoine, current studies are focussed on the

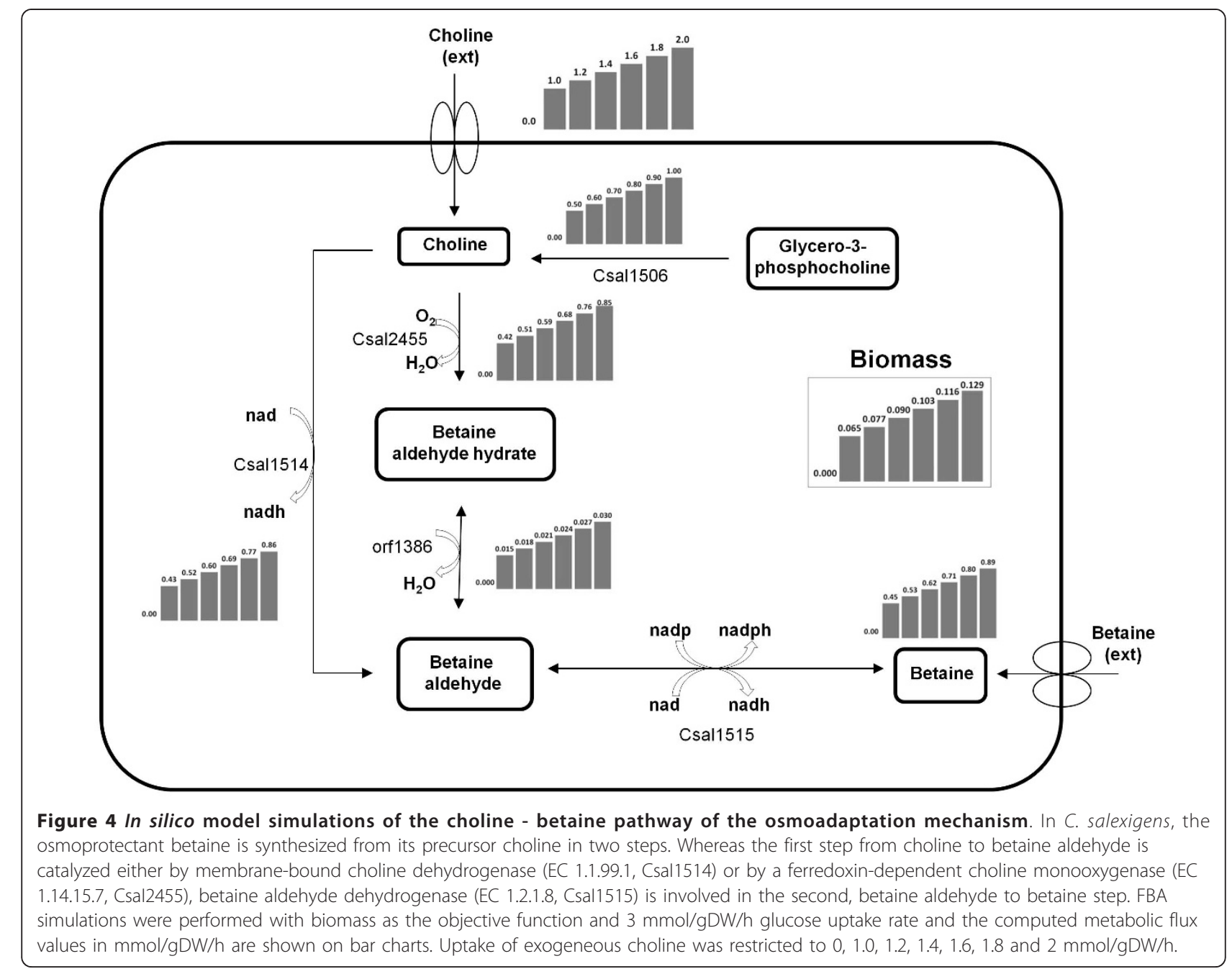


elucidation of its biosynthesis mechanism which in turn is essential for the improved production of this compatible solute. Ectoine is synthesized by $C$. salexigens in core osmoadaptation mechanism via ectABC genes (74). Its biosynthesis is a branch of the synthesis pathway for the aspartate family of amino acids (Figure 5). The aspartate is converted into aspartate- $\beta$-semialdehyde (ASA) via aspartate kinase (EC 2.7.2.4, Csal0626) and aspartate-semialdehyde dehydrogenase (EC 1.2.1.11, Csal2450), which is further converted to L-2,4-diaminobutyrate (DA) by diaminobutyrate-2-oxoglutarate transaminase (EC 2.6.1.76, Csal1877) requiring glutamate and by diaminobutyratepyruvate transaminase (EC 2.6.1.46, Csal1877) in the presence of alanine. L-2,4-diaminobutyrate is acetylated by DA acetyltransferase (EC 2.3.1.178, Csal1876) to $\mathrm{N} \gamma$ acetyl-L-2,4-diaminobutyrate (NADA), which is the substrate of ectoine synthase (EC 4.2.1.108, Csal1878).

To simulate metabolic model in the view of ectoine synthesis, the required conditions were implemented and the resulting flux values were investigated. To demonstrate high-level ectoine production when the other external osmoprotectants are not accessible, as stated by Vargas and co-workers (2008); under the absence of exogenous osmoprotectants (i.e. choline and betaine uptake as well as choline oxidation fluxes were constrained to zero), FBA simulations were performed for 3-10 $\mathrm{mmol} / \mathrm{gDW} / \mathrm{h}$ glucose uptake rates. Ectoine production increased from 1.4975 up to $4.9722 \mathrm{mmol} /$ $\mathrm{gDW} / \mathrm{h}$ with a yield within a range of $49-50 \% \mathrm{mmol}$ ectoine $/ \mathrm{mmol}$ glucose with concomitant increase in biomass $\left(0.1934\right.$ to $\left.0.642 \mathrm{~h}^{-1}\right)$ demonstrating the high level ectoine production when glucose was the only carbon source. In addition, Fallet and coworkers (2010) reported batch cultivation data for ectoine production with glucose as the sole carbon source. The performed FBA simulations with $1.5 \mathrm{mmol} / \mathrm{gDW} / \mathrm{h}$ glucose uptake resulted in an ectoine production rate of $0.75 \mathrm{mmol} /$ $\mathrm{gDW} / \mathrm{h}$, which was comparable with the reported experimental result of $0.72 \mathrm{mmol} / \mathrm{gDW} / \mathrm{h}$ [70].

Comprehensive analysis of the ectoine biosynthesis (Figure 5) revealed the importance of aspartate, glutamate and alanine in directing fluxes through ectoine synthesis pathway. Moreover, key enzymes of the pathway (i.e. aspartate kinase, diaminobutyrate-2-oxoglutarate transaminase, diaminobutyrate-pyruvate transaminase and DA acetyltransferase) link the pathway to the central metabolism. In FBA simulations, the presence of glutamate and alanine in the medium significantly affected both growth and ectoine production. For instance, constraining the glucose and $\mathrm{NaCl}$ uptake rates at $1 \mathrm{mmol} /$

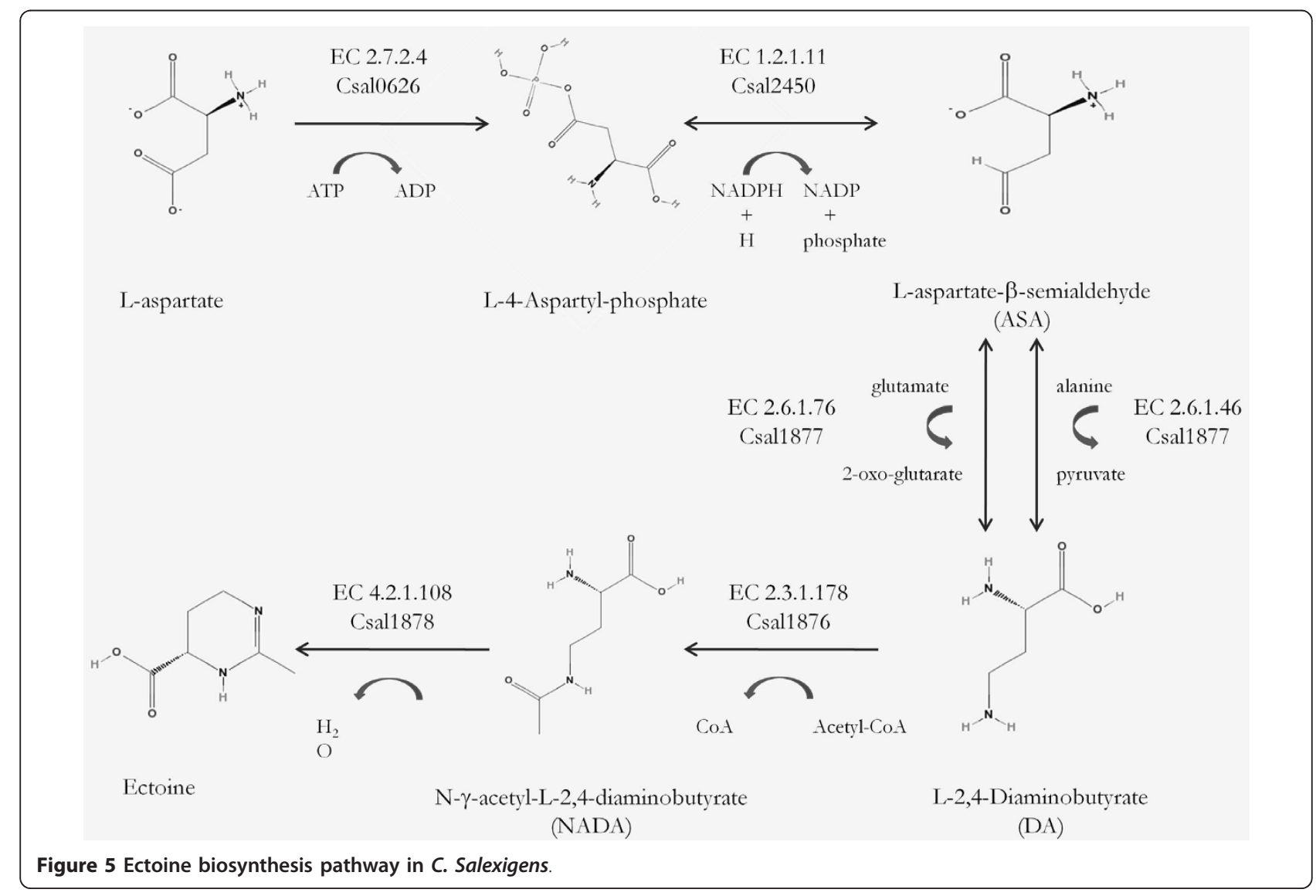


$\mathrm{gDW} / \mathrm{h}$ and $1.1 \mathrm{mmol} / \mathrm{gDW} / \mathrm{h}$, respectively; the presence of alanine in the medium was simulated by an uptake rate of $1.2 \mathrm{mmol} / \mathrm{gDW} / \mathrm{h}$ and the growth was stimulated by $9.01 \%$ (from 0.0710 to $0.0774 \mathrm{~h}^{-1}$ ), whereas the ectoine production was improved $9.08 \%$ (from 0.5497 to 0.5996 $\mathrm{mmol} / \mathrm{gDW} / \mathrm{h})$.

\section{Conclusions}

A non-automated but iterative decision-making process was employed in order to reconstruct the first comprehensive genome-scale metabolic model of a halophilic bacterium, C. salexigens DSM 3043. The in silico model was able not only to represent the potential of the network in terms of phenotypic characterization but also to predict metabolic fluxes during osmoadaptation, both of which were consistent with the experimental observations. The reconstructed model will accelarate the research on halophilic bacteria towards application of systems biology approaches, design of optimal culture conditions and metabolic engineering strategies for improved production of biological and industrially important products.

\section{Additional material}

Additional file 1: The metabolite and reaction lists of $C$. salexigens iOA584 metabolic model (Excel file).

Additional file 2: The complete list of genes and their annotations related to the transport mechanisms of C. salexigens DSM 3043 (Excel file).

Additional file 3: Topological analysis of the reconstructed $C$. salexigens iOA584 metabolic model (Excel file).

Additional file 4: The overall network map and detailed batch images of C. salexigens iOA584 metabolic model (Word document).

\section{Acknowledgements}

This research has been supported by Turkish State Planning Organization (DPT) through grant no, DPT09K120520 and TUBITAK MAG/110M613. Fellowship of Ozlem Ates provided by Italian and Turkish governments through TBAG-U/192(106T756) is gratefully acknowledged.

\section{Authors' contributions}

ETO and KYA conceived and directed the study. OA and KYA designed the algorithms and the reconstruction framework. OA performed the reconstruction process, analyzed the data and evaluated the model. OA, ETO and KYA wrote the paper. All authors read and approved the final version.

Received: 4 June 2010 Accepted: 21 January 2011

Published: 21 January 2011

\section{References}

1. Chien A, Edgar DB, Trela JM: Deoxyribonucleic acid polymerase from the extreme thermophile Thermus aquaticus. J Bacteriol 1976, 127:1550-1557.

2. Bartlett JMS, Stirling D: A Short History of Polymerase Chain Reaction. In Methods in Molecular Biology. In PCR Protocols. Volume 226. 2 edition. Edited by: Bartlett JMS, Stirling D. Humana Press; 2003:3-6.

3. Podar M, Reysenbach : New opportunities revealed by biotechnological explorations of extremophiles. Curr Opin Biotechnol 2006, 17:250-255.
4. Oren A: Diversity of halophilic microorganisms: environments, phylogeny, physiology, and applications. J Ind Microbiol Biotechnol 2002, 28:56-63.

5. Canovas D, Vargas C, Csonka LN, Ventosa A, Nieto Jj: Osmoprotectants in Halomonas elongata: high-affinity betaine transport system and cholinebetaine pathway. J Bacteriol 1996, 178:221-226.

6. Arahal D, Garcia MT, Vargas C, Canovas D, Nieto JJ, Ventosa A: Chromohalobacter salexigens sp. nov., a moderately halophilic species that includes Halomonas elongata DSM 3043 and ATCC 33174. Int J Syst Evol Microbiol 2001, 50:1457-1462.

7. Ventosa A, Nieto JJ, Oren A: Biology of moderately halophilic aerobic bacteria. Microbiol Mol Biol Rev 1998, 62:504-544.

8. Vargas C, Argandoña M, Reina-Bueno M, Rodriguez-Moya J, FernándezAunión C, Nieto JJ: Unravelling the adaptation responses to osmotic and temperature stress in Chromohalobacter salexigens, a bacterium with broad salinity tolerance. Saline Syst 2008, 4:14.

9. Argandoña M, Nieto JJ, Iglesias-Guerra F, Calderón Ml, García-Estepa R, Vargas C: Interplay between iron homeostasis and the osmostress response in the halophilic bacterium Chromohalobacter salexigens. Appl Environ Microbiol 2010, 76(11):3575-3589.

10. Ventosa A, Nieto Jj: Biotechnological applications and potentialities of halophilic microorganisms. World J Microb Biotechnol 1995, 11:85-94.

11. Klenk HP, Clayton RA, Tomb JF, et al: The complete genome sequence of the hyperthermophilic, sulphate-reducing archaeon Archaeoglobus fulgidus. Nature 1997, 390:364-370.

12. WV Ng, Kennedy SP, Mahairas GG, Berquist B, et al: Genome sequence of Halobacterium species NRC- 1. Proc Natl Acad Sci USA 2000, 97:12176-1218

13. Barreto M, Valdes J, Dominguez C, Arriagada C, Silver S, Bueno S, Jedlicki E, Holmes D: Whole Genome Sequence of Acidithiobacillus ferrooxidans: Metabolic Reconstruction, Heavy Metal Resistance and Other Characteristics. In Biohydrometallurgy: Fundamentals, Technology and Sustainable Development Minas Gerais, Brazil, Elsevier Press; 2001, 237-251.

14. The Joint Genome Institue. [http://genome.jgi-psf.org/].

15. Vargas C, Nieto JJ: Genetic tools for the manipulation of moderately halophilic bacteria of the family Halomonadaceae. In Methods in Molecular Biology, Recombinant Gene Expression: Reviews and Protocols. Volume 267. 2 edition. Edited by: Balbas P, Lorence A. Totowa, NJ, Humana Press; 2004:183-208.

16. Oren A, Larimer F, Richardson P, Lapidus A, Csonka LN: How to be moderately halophilic with broad salt tolerance: clues from the genome of Chromohalobacter salexigens. Extremophiles 2005, 9:275-279.

17. Oberhardt MA, Palsson BO, Papin JA: Applications of genome-scale metabolic reconstructions. Mol Syst Biol 2009, 5:320.

18. Feist AM, Hergard MJ, Thiele I, Reed JL, Palsson BO: Reconstruction of biochemical networks in microorganisms. Nat Rev Microbiol 2009, 7:129-143

19. Durot M, Bourguignon PY, Schachter V: Genome-scale models of bacterial metabolism: reconstruction and applications. FEMS Microbial Rev 2009, 33:164-190,

20. Francke C, Siezen RJ, Teusink B: Reconstructing the metabolic network of a bacterium from its genome. Trends Microbiol 2005, 13:550-558.

21. Herrgård MJ, Swainston N, Dobson P, Dunn WB, Arga KY, et al: A consensus yeast metabolic network reconstruction obtained from a community approach to systems biology. Nat Biotechno 2008, 26:1155-1160.

22. Notebaart RA, van Enckevort FH, Franche C, Siezen RJ, Teusink B: Accelerating the reconstruction of genome-scale metabolic networks. BMC Bioinformatics 2006, 7:296.

23. Thiele I, Palsson BO: A protocol for generating a high-quality genomescale metabolic reconstruction. Nature Protocols 2010, 5:93-121.

24. Karp PD, Paley S, Romero P: The pathway tools software. Bioinformatics 2002, 18(Suppl 1):S225-S232.

25. Pinney JW, Shirley MW, McConkey GA, Westhead DR: metaSHARK: software for automated metabolic network prediction from DNA sequence and its application to the genomes of Plasmodium falciparum and Eimeria tenella. Nucl Acids Res 2005, 33(4):1399-1409.

26. Reed JL, Famili I, Thiele I, Palsson BO: Towards multidimensional genome annotation. Nat Reviews Genet 2006, 7:130-141. 
27. Forster J, Famili I, Fu P, Palsson BO, Nielsen J: Genome-scale reconstruction of the Saccharomyces cerevisiae metabolic network. Genome Res 2003, 13:244-253.

28. Duarte NC, Herrgard MJ, Palsson BO: Reconstruction and validation of Saccharomyces cerevisiae iND750, a fully compartmentalized genomescale metabolic model. Genome Res 2004, 14:1298-1309.

29. Nookaew I, Jewett MC, Meechai A, Thammarongtham C, Laoteng K, Cheevadhanarak S, Nielsen J, Bhumiratana S: The genome-scale metabolic model ilN800 of Saccharomyces cerevisiae and its validation: a scaffold to query lipid metabolism. BMC Syst Biol 2008, 2:71.

30. Duarte NC, Becker SA, Jamshidi N, Thiele I, Mo ML, Vo TD, Srivas R, Palsson BO: Global reconstruction of the human metabolic network based on genomic and bibliomic data. Proc Natl Acad Sci USA 2007, 104:1777-1782.

31. Radrich K, Tsuruoka Y, Dobson P, Gevorgan A, Swainston N, Baart G, Schwartz JM: Integration of metabolic databases for the reconstruction of genome-scale metabolic networks. BMC Systems Biology 2010, 4:114.

32. Edwards JS, Palsson BO: The Escherichia coli MG1655 in silico metabolic genotype: its definition, characteristics, and capabilities. Proc Natl Acad Sci USA 2000, 97:5528-5533.

33. Reed $J$, Vo TD, Schilling CH, Palsson BO: An expanded genome-scale model of Escherichia coli K-12 (iJR904 GSM/GPR). Genome Biol 2003, 4 R54.1-R54.12.

34. Feist AM, Henry CS, Reed JL, Krummenacker M, Joyce AR, Karp PD, Broadbelt LJ, Hatzimanikatis V, Palsson BO: A genome-scale metabolic reconstruction for Escherichia coli K-12 MG1655 that accounts for 1260 ORFs and thermodynamic information. Mol Syst Biol 2007, 3:121.

35. Oh YK, Palsson BO, Park SM, Schilling CH, Mahadevan R: Genome-scale reconstruction of metabolic network in Bacillus subtilis based on highthroughput phenotyping and gene essentialitydata. J Biol Chem 2007, 282(39):28791-9.

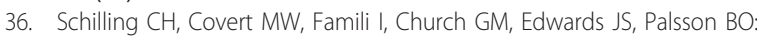
Genome-scale metabolic model of Helicobacter pylori 26695. J Bacteriol 2002, 184:4582-4593.

37. Thiele I, Vo TD, Price ND, Palsson BO: Expanded metabolic Reconstruction of Helicobacter pylori (ilT341 GSM/GPR): an In Silico Genome-Scale Characterization of Single- and Double-Deletion Mutants. J Bacterio/ 2005, 187:5818-5830.

38. Oliveira AP, Nielsen J, Forster J: Modeling Lactococcus lactis using a genome-scale flux model. BMC Microbiol 2005, 5:39.

39. Becker S, Palsson BO: Genome-scale reconstruction of the metabolic network in Staphylococcus aureus N315: an initial draft to the twodimensional annotation. BMC Microbiol 2005, 5:8.

40. Lee DS, Burd H, Liu J, Almaas E, Wiest O, Barabasi AL, Oltvai ZN, Kapatral V Comparative genome-scale metabolic reconstruction and flux balance analysis of multiple Staphylococcus aureus genomes identify novel antimicrobial drug targets. J Bacterio/ 2009, 191:4015-4024.

41. Lee J, Yun H, Feist A, Palsson BO, Lee S: Genome-scale reconstruction and in silico analysis of the Clostridium acetobutylicum ATCC 824 metabolic network. Appl Microbiol Biot 2008, 80:849-862.

42. Nogales J, Palsson BO, Thiele I: A genome-scale metabolic reconstruction of Pseudomonas putida KT2440: iJN746 as a cell factory. BMC Syst Biol 2008, 2:79.

43. Oberhardt MA, Puchalka J, Fryer KE, Martins dos Santos VAP, Papin JA: Genome-Scale Metabolic Network Analysis of the Opportunistic Pathogen Pseudomonas aeruginosa PAO1. J Bacteriol 2008, 190(8):2790-2803.

44. Sun J, Sayyar B, Butler JE, Pharkya P, Fahland TR, Famili I, Schilling $\mathrm{CH}$, Lovley DR, Mahadevan R: Genome-scale constraint-based modeling of Geobacter metallireducens. BMC Syst Biol 2009, 3:15.

45. Kjeldsen KR, Nielsen J: In silico genome-scale reconstruction and validation of the Corynebacterium glutamicum metabolic network. Biotechnol Bioeng 2009, 102(2):583-97.

46. Feist AM, Scholten JC, Palsson BO, Brockman FJ, Ideker T: Modeling methanogenesis with a genome-scale metabolic reconstruction of Methanosarcina barkeri. Mol Syst Biol 2006, 2, 2006.004.

47. Gonzalez O, Gronau S, Falb M, Pfeiffer F, Mendoza E, Zimmer R, Oesterhelt D: Reconstruction, modeling \& analysis of Halobacterium salinarum R-1 metabolism. Mol Biosyst 2008, 4:148-159.

48. Computational Biology at ORNL. [http://genome.ornl.gov/microbial/csal], assembled 08.Nov.2005.
49. DOE Joint Genome Institute. [http://img.jgi.doe.gov/cgi-bin/pub/main.cgi]

50. Kanehisa M, Goto S, Furumichi M, Tanabe M, Hirakawa M: KEGG for representation and analysis of molecular networks involving diseases and drugs. Nucleic Acids Res 2010, 38:D355-D360.

51. Schellenberger J, Park JO, Conrad TC, Palsson BO: BiGG: a Biochemical Genetic and Genomic knowledgebase of large scale metabolic reconstructions. BMC Bioinformatics 2010, 11:213.

52. Gasteiger E, Gattiker A, Hoogland C, Ivanyi I, Appel RD, Bairoch A: ExPASy: the proteomics server for in-depth protein knowledge and analysis. Nucleic Acids Res 2003, 31:3784-3788.

53. Karp PD, Ouzounis CA, Moore-Kochlacs C, Goldovsky L, Kaipa P, Ahren D, Tsoka S, Darzentas N, Kunin V, Lopez-Bigas N: Expansion of the BioCyc collection of pathway/genome databases to 160 genomes. Nucleic Acids Res 2005, 19:6083-89.

54. Chang A, Scheer M, Grote A, Schomburg I, Schomburg D: BRENDA, AMENDA and FRENDA the enzyme information system: new content and tools in 2009. Nucleic Acids Res 2009, 37:D588-D592.

55. Palsson BO: Systems biology: Properties of reconstructed networks Cambridge University Press, New York; 2006.

56. Arga KY, Önsan Zi், Kırdar B, Ülgen KÖ, Nielsen J: Understanding signaling in yeast: insights from network analysis. Biotechnol Bioeng 2007 97(5):1246-1258

57. Becker SA, Price ND, Palsson BO: Metabolite coupling in genome-scale metabolic networks. BMC Bioinf 2006, 7:111

58. Canovas D, Vargas C, Csonka LN, Ventosa A, Nieto JJ: Synthesis of glycine betaine from exogenous choline in the moderately halophilic bacterium Halomonas elongate. Environ Microbiol 1998, 64:4095-4097, Appl.

59. Vargas C, Jebbar M, Carrasco R, Blanco C, Calderon MI, Iglesias-Guerra F, Nieto JJ: Ectoines as compatible solutes and carbon and energy sources for the halophilic bacterium Chromohalobacter salexigens. J Appl Microbiol 2006, 100:98-107.

60. Schilling $\mathrm{CH}$, Schuster S, Palsson BO, Heinrich R: Metabolic pathway analysis: Basic concepts and scientific applications in the post-genomic era. Biotechnol Prog 1999, 15:296-303.

61. Lee JM, Gianchandani EP, Papin JA: Flux balance analysis in the era of metabolomics. Brief Bioinf 2006, 7:140-150.

62. Raman K, Chandra N: Flux balance analysis of biological systems: applications and challenges. Briefings Bioinf 2009, 10:435-449.

63. Khannapho C, Zhao H, Bonde BK, Kierzek AM, Avignone-Rossa CA, Bushell ME: Selection of objective function in genome scale flux balance analysis for process feed development in antibiotic production. Metab Eng 2008, 10(5):227-233.

64. Puchałka J, Oberhardt MA, Godinho M, Bielecka A, Regenhardt D, Timmis KN, Papin JA, Martins dos Santos VAP: Genome-Scale Reconstruction and Analysis of the Pseudomonas putida KT2440 Metabolic Network Facilitates Applications in Biotechnology. PLoS Comput Biol 2008, 4(10):e1000210.

65. Mahadevan $\mathrm{R}$, Schilling $\mathrm{CH}$ : The effects of alternate optimal solutions in constraints-based genome scale metabolic models. Met Eng 2003, 5:264-276.

66. Uchiyama I, Higuchi T, Kawai M: MBGD update 2010: toward a comprehensive resource for exploring microbial genome diversity. Nucleic Acids Research 2010, 38:D361-D365.

67. de la Haba RR, Arahal DR, Marquez MC, Ventosa A: Phylogenetic relationships within the family Halomonadaceae based on comparative $23 \mathrm{~S}$ and 16S rRNA gene sequence analysis. Int J Syst Evol Microbiol 2010, 60:737-748.

68. Clemente JC, Satou K, Valiente G: Finding conserved and non-conserved reactions using a metabolic pathway alignment algorithm. Genome Inf 2006, 17(2):46-56.

69. Machielsen R, Looger LL, Raedts J, Dijkhuizen S, Hummel W, Hennemann HG, Daussmann T, van der Oost J: Cofactor engineering of Lactobacillus brevis alcohol dehydrogenase by computational design. Eng Life Sci 2009, 9(1):38-44.

70. Fallet C, Rohe P, Franco-Lara E: Process optimization of the integrated synthesis and secretion of ectoine and hydroxyectoine under hyper/ hypo-osmotic strees. Biotechnol Bioeng 2010, 107:124-133.

71. Schubert T, Maskow T, Benndorf D, Harms H, Breuer U: Continuous synthesis and excretion of the compatible solute ectoine by a transgenic, nonhalophilic bacterium. Appl Environ Microbiol 2007 73:3343-3347. 
72. Empadinhas N, da Costa MS: Osmoadaptation mechanisms in prokaryotes: distribution of compatible solutes. Int Microbiol 2008, 11:151-161.

73. Galinski EA: Osmoadaption in bacteria. Advances in Microbial Physiology 1995, 37:273-328.

74. Calderón MI, Vargas C, Rojo F, Iglesias-Guerra F, Csonka LN, Ventosa A, Nieto JJ: Complex regulation of the synthesis of the compatible solute ectoine in the halophilic bacterium Chromohalobacter salexigens DSM 3043. Microbiology. 2004, 150:3051-3063.

doi:10.1186/1752-0509-5-12

Cite this article as: Ates et al: Genome-scale reconstruction of metabolic network for a halophilic extremophile, Chromohalobacter salexigens DSM 3043. BMC Systems Biology 2011 5:12.

\section{Submit your next manuscript to BioMed Central} and take full advantage of:

- Convenient online submission

- Thorough peer review

- No space constraints or color figure charges

- Immediate publication on acceptance

- Inclusion in PubMed, CAS, Scopus and Google Scholar

- Research which is freely available for redistribution

Submit your manuscript at www.biomedcentral.com/submit
() Biomed Central 